\title{
Diffraction and reflection of antiplane shear waves in a cracked couple stress elastic material
}

\author{
Andrea Nobili ${ }^{1}$, Enrico Radi ${ }^{2}$, Gennady Mishuris ${ }^{3}$ \\ ${ }^{1}$ Dipartimento di Ingegneria Enzo Ferrari, Università di Modena e Reggio Emilia, via Vivarelli 10, \\ 41125 Modena, Italy \\ E-mail:andrea.nobili@unimore.it \\ 2 Dipartimento di Scienze e Metodi dell'Ingegneria, Università di Modena e Reggio Emilia, via \\ Amendola 2, 42122 Reggio Emilia, Italy \\ E-mail:enrico.radi@unimore.it \\ ${ }^{3}$ Institute of Mathematical and Physical Sciences, Aberystwyth University, Ceredigion SY23 3BZ, \\ Wales, UK \\ E-mail:ggm@aber.ac.uk
}

Keywords: Couple stress; Wave diffraction; Rayleigh waves

We investigate the effect of a semi-infinite rectilinear crack on diffraction and reflection of antiplane shear waves in an elastic solid with microstructure. Waves are induced by moving shear traction vectors applied at the faces of the crack. The material behavior is described by the indeterminate theory of couple stress elasticity considering micro inertia. This elastic constitutive model accounts for the material microstructure and it is a special case of the micropolar theory; it was developed by Koiter [3] for the quasi-static regime and later extended by Eringen [1] to include dynamic effects. The full-field solution is obtained through integral transforms and the Wiener-Hopf technique [5] and it may be used as a bulding block to solve general wave propagation problems in a cracked half-space in antiplane deformation. The solution differs significantly from the classical result given in [2] for isotropic elastic materials. Indeed, unlike classical elasticity, antiplane shear Rayleigh waves are supported in couple stress materials [4]. A complicated wave pattern appears which consists of entrained waves extending away from the crack, reflected Rayleigh waves, localized waves irradiating from and body waves scattered by the crack-tip. Wave diffraction and interference brings an important contribution to the stress intensity factors originally presented in [6] in the static framework. Resonance is triggered when the applied loading is fed into the crack-tip at Rayleigh speed and this result is confirmed by the evaluation of the energy release rate.

References

[1] A. C. Eringen, "Theory of micropolar elasticity". In Microcontinuum field theories, pages 101-248. Springer, 1999.

[2] L. B. Freund, Dynamic fracture mechanics. Cambridge Monographs on Mechanics and Applied Mathematics. Cambridge university press, Cambridge, 1990.

[3] W. Koiter, "Couple-stress in the theory of elasticity". In Proc. K. Ned. Akad. Wet, volume 67, pages 17-44. North Holland Pub, 1964.

[4] A. Nobili, E. Radi, and A. Vellender, "Diffraction of antiplane shear waves and stress concentration in a cracked couple stress elastic material with micro inertia". Journal of the Mechanics and Physics of Solids, 124:663 - 680, 2019.

[5] B. Noble, Methods based on the Wiener-Hopf technique for the solution of partial differential equations, International Series of Monographs on Pure and Applied Mathematics. Vol. 7. Pergamon Press, New York, 1958.

[6] E. Radi, "On the effects of characteristic lengths in bending and torsion on mode III crack in couple stress elasticity". International Journal of Solids and Structures, 45(10):3033-3058, 2008. 